\title{
A Novel Modified-T Equivalent Circuit for Modeling LTCC Embedded Inductors With a Large Bandwidth
}

\author{
Tzyy-Sheng Horng, Member, IEEE, Jian-Ming Wu, Li-Qun Yang, and Shyh-Tirng Fang, Member, IEEE
}

\begin{abstract}
This paper presents a new modified-T model for three-dimensional inductors embedded in the multilayer low-temperature cofired-ceramic substrate with a remarkably large bandwidth. This model combines a core circuit, a five-element modified-T model, to include the effects of a transmission line with an electrical length up to $\pi$, and several resonators to consider the effects due to coil coupling and the finite ground plane. It is emphasized that most of the elements in this equivalent-circuit model can be extracted directly from the measured $S$-parameters. Compared to the conventional PI-section model that is valid only up to the fundamental self resonant frequency (SRF), this model can include more higher-order SRFs and ground resonant frequencies effectively, which results in several times larger bandwidth.
\end{abstract}

Index Terms-Embedded inductor, inductor model, lowtemperature cofired-ceramic (LTCC) inductor, three-dimensional (3-D) inductor.

\section{INTRODUCTION}

$\mathbf{L}$ OW-TEMPERATURE cofired-ceramic (LTCC) embedded inductors can be viewed as passive interconnects that are conventionally modeled using PI- or T-section models [1]-[3]. Although several additional capacitances and resistances have been included to account for coupling and loss in the coil and substrate, these models are still narrow-banded due to the nature of PI- and T-section models that cannot represent a transmission line over a wide frequency range effectively. The distributed model using multiple PI- or T-sections can be applied to overcome this drawback [4], [5]. However, it is difficult to find these distributed elements from the measured $S$-parameters without using intensive optimization. This is because the measured $S$-parameters providing the overall frequency response for an equivalent distributed circuit lack useful information on the segmentation of the individual section.

This paper breaks through the above-mentioned modeling barriers by inventing a five-element modified-T circuit that can approximate very closely a lossless transmission line with electrical length up to $\pi$, the well-known period of transmission-line effects. In conjunction with several resonators, this modified-T circuit can be expanded to model real LTCC em-

Manuscript received April 17, 2003; revised July 18, 2003. This work was supported by the R.O.C. Ministry of Education Program for Promoting Academic Excellence of Universities under Grant 91-E-FA08-1-4.

T.-S. Horng and J.-M. Wu are with the Department of Electrical Engineering, National Sun Yat-Sen University, Kaohsiung 804, Taiwan, R.O.C. (e-mail: jason@ee.nsyu.edu.tw).

L.-Q. Yang and S.-T. Fang are with the Computer and Communications Research Laboratories, Industrial Technology Research Institute, Hsinchu 310 Taiwan, R.O.C. (e-mail: tonyfang@itri.org.tw).

Digital Object Identifier 10.1109/TMTT.2003.819772

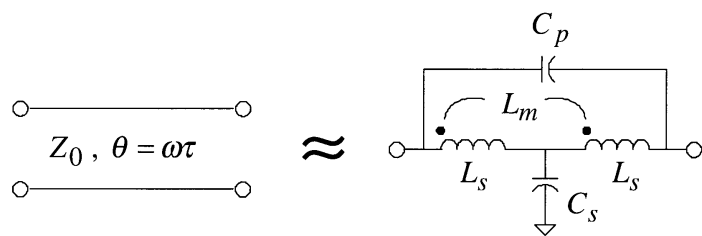

Fig. 1. Five-element modified-T equivalent circuit for modeling a lossless transmission line with electrical length up to $\pi$.

bedded inductors over a large bandwidth. Although such a modified-T equivalent circuit to be established needs quite a number of circuit elements, our modeling approach still shows amazing efficiency by skillfully formulating with all kinds of resonant frequencies in an element-extraction procedure. As a result, all the reactive elements in the model can be extracted from the measured $S$-parameters directly. In the demonstration, three different types of LTCC embedded inductors including spiral, meander, and vertically stacked spiral have been measured with two-port $S$-parameters from which both modified-T and PI-section equivalent circuits are established for comparison. The modified-T model is superior to the PI-section model quite obviously with a several times larger amount of modeling bandwidth.

\section{MOdIFIED-T MODEL FOR TRANSMISSION LINES}

In our previous work [6], we have demonstrated the mathematical extraction procedure to find the circuit elements in an equivalent modified-T model for ideal transmission lines, as shown in Fig. 1. The circuit elements can be expressed in terms of transmission-line parameters as

$$
\begin{aligned}
L_{s} & =Z_{0} \tau\left(\frac{1}{4}+\frac{1}{\pi^{2}}\right) \\
L_{m} & =Z_{0} \tau\left(\frac{1}{4}-\frac{1}{\pi^{2}}\right) \\
C_{s} & =\frac{\tau}{Z_{0}} \\
C_{p} & =\frac{\tau}{Z_{0} \pi^{2}} .
\end{aligned}
$$

For example, we compare the simulated two-port $S$-parameters from the transmission-line configuration shown in Fig. 2, and those from its equivalent modified-T model whose circuit elements can be found according to (1)-(4). Fig. 3 shows a comparison of these $S$-parameters on a Smith chart under various normalized characteristic impedances $\left(z_{0}\right)$ with a sweep of electrical length from zero to $\pi$. It can be seen that both $S$-parameter responses are almost identical. In Fig. 4, the mean and maximum absolute values for the difference of $S$-parameters in the 


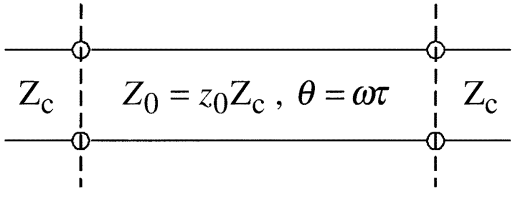

Fig. 2. Transmission-line configuration used for comparing its two-port $S$-parameters with those in an equivalent modified-T model. Assume $\tau=100 \mathrm{ps}$ for this case.

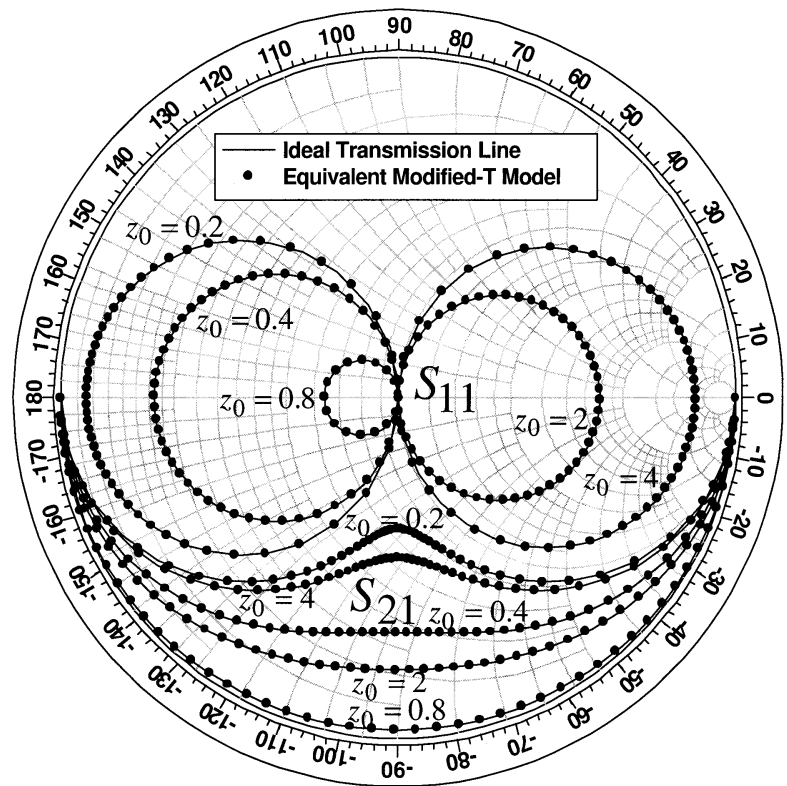

Fig. 3. Comparison of $S_{11}$ and $S_{21}$ on a Smith chart between the transmission-line circuit shown in Fig. 2 and its equivalent modified-T model with a sweep of electrical length from zero to $\pi$.

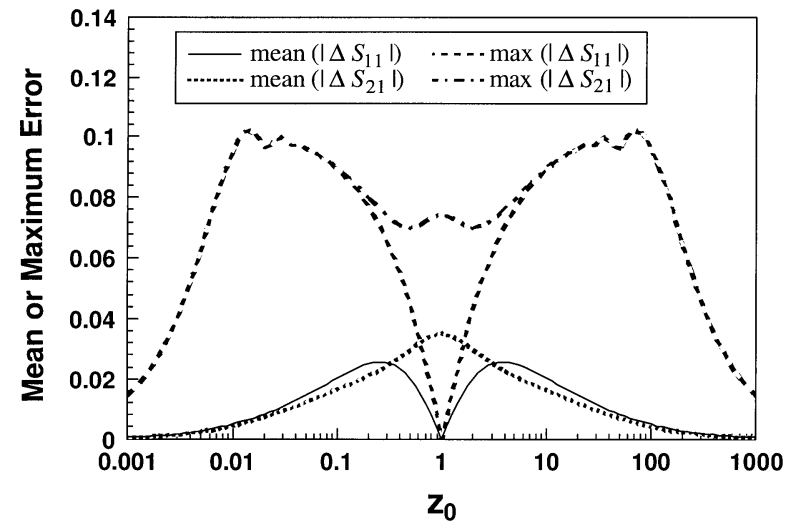

Fig. 4. Maximum and mean absolute values versus normalized characteristic impedance for the $S$-parameter differences between the transmission-line circuit shown in Fig. 2 and its equivalent modified-T model.

range of electrical length from zero to $\pi$ are shown as a function of $z_{0}$. One can see that the mean and maximum errors are less than 0.04 and 0.1 , respectively, for all possible values of $z_{0}$. It is, therefore, to be believed that the proposed modified-T model can very closely represent a lossless transmission-line section with a wide range of characteristic impedance and an electrical length up to $\pi$.

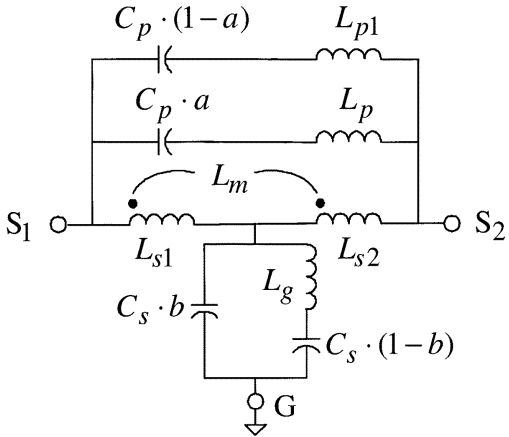

Fig. 5. Expanded modified-T equivalent circuit for modeling an LTCC embedded inductor.

\section{MODIFIED-T MODEL FOR LTCC EMBEDDED INDUCTORS}

As suggested in Fig. 5, the modified-T equivalent circuit can be further expanded to model the three-dimensional (3-D) inductors embedded in a multilayer LTCC substrate. Several $L C$ resonators have been added to account for the self and ground resonant phenomena. The formulation starts with a derivation of the corresponding $Y$ network parameters given as

$$
\begin{aligned}
Y_{11} & =\frac{j \omega L_{s 2}+Z_{g}}{-\omega^{2}\left(L_{s 1} L_{s 2}-L_{m}^{2}\right)+j \omega Z_{g}\left(L_{s 1}+L_{s 2}+2 L_{m}\right)}+Y_{p} \\
Y_{22} & =\frac{j \omega L_{s 1}+Z_{g}}{-\omega^{2}\left(L_{s 1} L_{s 2}-L_{m}^{2}\right)+j \omega Z_{g}\left(L_{s 1}+L_{s 2}+2 L_{m}\right)}+Y_{p} \\
Y_{12} & =Y_{21} \\
& =\frac{j \omega L_{m}-Z_{g}}{-\omega^{2}\left(L_{s 1} L_{s 2}-L_{m}^{2}\right)+j \omega Z_{g}\left(L_{s 1}+L_{s 2}+2 L_{m}\right)}-Y_{p}
\end{aligned}
$$

where

$$
\begin{aligned}
Z_{g}= & \left\{j \omega C_{s} b+\left\{j \omega L_{g}+\left[j \omega C_{s}(1-b)\right]^{-1}\right\}^{-1}\right\}^{-1} \\
Y_{p}= & \left\{j \omega L_{p}+\left(j \omega C_{p} a\right)^{-1}\right\}^{-1} \\
& +\left\{j \omega L_{p 1}+\left[j \omega C_{p}(1-a)\right]^{-1}\right\}^{-1} .
\end{aligned}
$$

Note that $Z_{g}$ denotes the series-feedback resonator impedance, while $Y_{P}$ denotes the total admittance for all parallel-feedback resonators. In the extraction of circuit elements, we use the real $S$-parameter measurement results of a 6.8-nH LTCC embedded inductor to illustrate that the whole procedure can be carried out efficiently and reliably in the following steps.

\section{Step 1. Formulation With Parallel and Series Resonant Frequencies}

First, a new parameter $Y_{A}$ composed of $Y$ network parameters is defined as

$$
\begin{aligned}
Y_{A} & =Y_{11}+Y_{22}-2 Y_{12} \\
& =\frac{j \omega\left(L_{s 1}+L_{s 2}-2 L_{m}\right)+4 Z_{g}}{-\omega^{2}\left(L_{s 1} L_{s 2}-L_{m}^{2}\right)+j \omega Z_{g}\left(L_{s 1}+L_{s 2}+2 L_{m}\right)}+4 Y_{p} .
\end{aligned}
$$




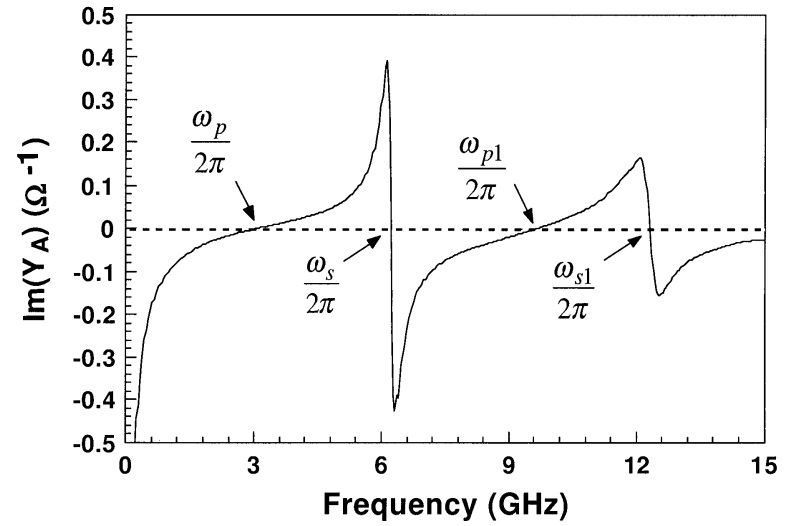

Fig. 6. Determination of parallel and series resonant frequencies from the imaginary part of $Y_{A}$.

Assuming $L_{s 1} \approx L_{s 2}$ and simplifying (10) gives

$$
Y_{A} \approx \frac{4}{j \omega\left(L_{s 1}^{\prime}+L_{s 2}^{\prime}\right)}+4 Y_{p}
$$

where

$$
\begin{aligned}
& L_{s 1}^{\prime}=L_{s 1}+L_{m} \\
& L_{s 2}^{\prime}=L_{s 2}+L_{m} .
\end{aligned}
$$

One can see from (11) that such a new parameter can get rid of the factor of ground resonator impedance $\left(Z_{g}\right)$ under the assumption $L_{s 1} \approx L_{s 2}$. According to our observation, (11) is also often true even if $L_{s 1}$ is not so close to $L_{s 2}$. This is because $Z_{g}$ usually behaves as the dominant order in the fractional term of (10) and can be factored out finally. The sum value $\left(L_{s 1}^{\prime}+L_{s 2}^{\prime}\right)$ that appears in (11) represents the total inductance of the inductor. Generally speaking, this value can be extracted from the equivalent PI-section model quite robustly at low frequencies. Therefore, the information coming from $Y_{A}$ is mainly related to the parallel-feedback resonators in parallel connection with the total inductance, which will include multiple parallel and series resonances. Fig. 6 shows the imaginary part of the $Y_{A}$-parameter that can be obtained using conversion from $S$ into $Y$ network parameters. One can find two zeros and two poles within the measurement frequency range. The root positions identify the first and second parallel resonant frequencies $\omega_{p} / 2 \pi$ and $\omega_{p 1} / 2 \pi$, while the pole positions identify the first and second series resonant frequencies $\omega_{s} / 2 \pi$ and $\omega_{s 1} / 2 \pi$. It is noted that one pair of parallel and series resonances in the $Y_{A}$-parameter contributes to a self-resonance (transmission zero) in the $S_{21}$-parameter with a self resonant frequency (SRF) in between the parallel and series resonant frequencies. When formulated with these four identified resonant frequencies, the circuit elements in the parallel-feedback resonators can be expressed as

$$
\begin{aligned}
& C_{p}=\left\{( L _ { s 1 } ^ { \prime } + L _ { s 2 } ^ { \prime } ) \left[a\left(\omega_{p}^{-2}-\omega_{s}^{-2}\right)^{-1}\right.\right.\left.\left.+(1-a)\left(\omega_{p}^{-2}-\omega_{s 1}^{-2}\right)^{-1}\right]\right\}^{-1} \\
& L_{p}=\left(a \omega_{s}^{2} C_{p}\right)^{-1} \\
& L_{p 1}=\left[(1-a) \omega_{s 1}^{2} C_{p}\right]^{-1}
\end{aligned}
$$

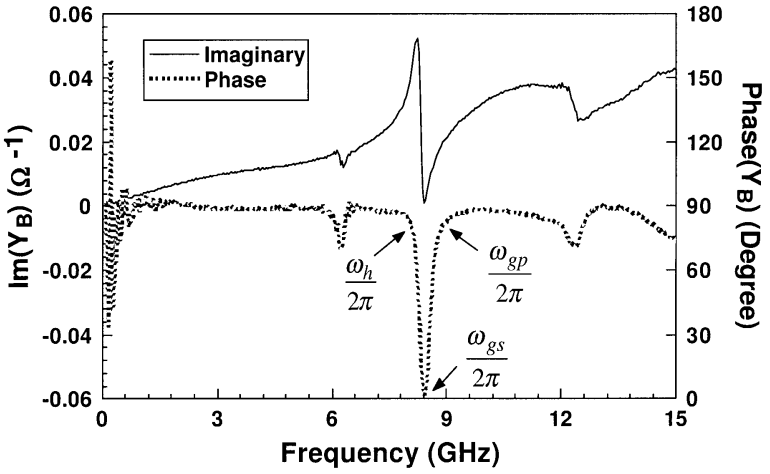

Fig. 7. Determination of half-wave frequencies and GRFs from the imaginary part or phase of $Y_{B}$.

where parameter $a$ in (14)-(16) represents a split coefficient for the parallel-feedback resonator capacitance and can be found as shown in (17) at the bottom of the following page.

\section{Step 2. Formulation With Half-Wave and Ground Resonant Frequencies (GRFs)}

Another new parameter, i.e., $Y_{B}$, also composed of $Y$ network parameters is defined as

$$
\begin{aligned}
Y_{B} & =Y_{11}+Y_{22}+2 Y_{12} \\
& =\frac{j \omega\left(L_{s 1}^{\prime}+L_{s 2}^{\prime}\right)}{-\omega^{2}\left[L_{s 1}^{\prime} L_{s 2}^{\prime}-L_{m}\left(L_{s 1}^{\prime}+L_{s 2}^{\prime}\right)\right]+j \omega Z_{g}\left(L_{s 1}^{\prime}+L_{s 2}^{\prime}\right)} .
\end{aligned}
$$

The purpose of using the $Y_{B}$-parameter is to remove the factor of $Y_{p}$, but maintain the factor of $Z_{g}$ instead. Therefore, the information coming from $Y_{B}$ is closely related to the series-feedback resonator, which will include the complete resonant effects due to the finite ground plane in an LTCC structure. Besides, the denominator in the expression of $Y_{B}$ goes to zero at the half-wave frequency $\left(\omega_{h} / 2 \pi\right)$. The reason to call it half-wave frequency is because for an ideal transmission line such as a pole position, which corresponds to the frequency at which the transmission line is a half-wavelength long [6]. Fig. 7 shows the imaginary part and also the phase for the $Y_{B}$-parameter. From the frequency response of the imaginary part, one can clearly see a dominant pole that locates at the half-wave frequency. More information can be found from the phase data. In the vicinity of a half-wave frequency, the phase has a sudden drop from almost $90^{\circ}$ to $0^{\circ}$. This is because two GRFs come after the half-wave frequency. The first one is the series GRF $\left(\omega_{g s} / 2 \pi\right)$, at which $Z_{g}$ approaches zero. The second one is the parallel GRF $\left(\omega_{g p} / 2 \pi\right)$, at which $Z_{g}$ approaches infinity. We can locate these frequencies according to the suggestions shown in Fig. 7. With these three newly identified half-wave frequencies and GRFs, we can formulate the mutual and ground inductances in the forms

$$
\begin{aligned}
L_{m} & =\frac{L_{s 1}^{\prime} L_{s 2}^{\prime}}{L_{s 1}^{\prime}+L_{s 2}^{\prime}}-\left\{\omega_{h}^{2} C_{s} b\left[1-\frac{(1-b) \omega_{g p}^{2}}{\omega_{h}^{2}-b \omega_{g p}^{2}}\right]\right\}^{-1} \\
L_{g} & =\left[\omega_{g p}^{2} C_{s} b(1-b)\right]^{-1}
\end{aligned}
$$




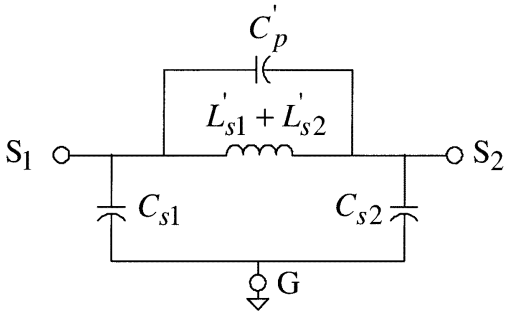

Fig. 8. Conventional PI-section model for inductors.

where the parameter $b$ in (19) and (20) represents another split coefficient for the series-feedback resonator capacitance and can be found as

$$
b=\frac{\omega_{g s}^{2}}{\omega_{g p}^{2}}
$$

Calculations of mutual and ground inductances in (19) and (20) require knowledge of the parameters $L_{s 1}^{\prime}, L_{s 2}^{\prime}$, and $C_{s}$ whose quantities can be extracted at low frequencies, as will be presented in the following step. It is also noted that there is an alternative way to accurately find the capacitance split coefficients $a$ and $b$ through optimization with the constrains

$$
\begin{aligned}
& 0 \leq a \leq 1 \\
& 0 \leq b \leq 1
\end{aligned}
$$

\section{Step 3. Extraction of Fundamental Elements at Low} Frequencies Using PI-Section Model

The well-known PI-section model, as shown in Fig. 8, can be used to extract some fundamental elements including the main inductance and shunt capacitance in a modified-T model. The extraction summarized as follows is mainly done at low frequencies:

$$
\begin{aligned}
L_{s 1}^{\prime}+L_{s 2}^{\prime} & \approx \frac{1}{\omega \operatorname{Im}\left\{Y_{12}\right\}} \\
\frac{L_{s 1}^{\prime}}{L_{s 2}^{\prime}} & \approx \frac{\operatorname{Im}\left\{Z_{11}-Z_{12}\right\}}{\operatorname{Im}\left\{Z_{22}-Z_{12}\right\}} \\
C_{s} & =C_{s 1}+C_{s 2} \approx \frac{\operatorname{Im}\left\{Y_{B}\right\}}{\omega} \\
\frac{C_{s 1}}{C_{s 2}} & \approx \frac{\operatorname{Im}\left\{Y_{11}+Y_{12}\right\}}{\operatorname{Im}\left\{Y_{22}+Y_{12}\right\}} .
\end{aligned}
$$

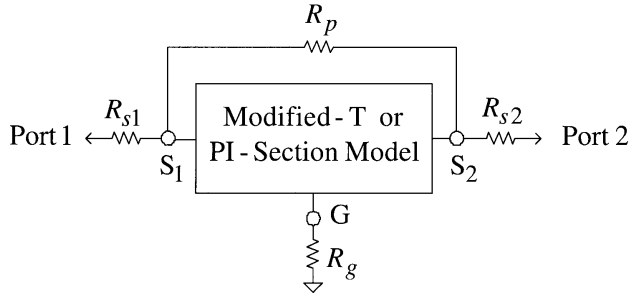

Fig. 9. Resistances added to the modified-T or PI-section models for inclusion of the frequency-dependent losses.

The parallel capacitance $C_{p}^{\prime}$ in an equivalent PI-section model is calculated as

$$
C_{p}^{\prime}=\frac{\omega_{r}^{-2}}{\left(L_{s 1}^{\prime}+L_{s 2}^{\prime}\right)}
$$

where $\omega_{r}$ is found from $\operatorname{lm}\left\{Y_{12}\left(\omega_{r}\right)\right\}=0$. After this step, we have found all the reactive elements in the equivalent modified-T and PI-section models analytically.

\section{Step 4. Optimization for Resistance Values According to Frequency-Dependent Quality (Q) Factor}

Some resistances are included in the equivalent modified-T or PI-section models to account for the frequency-dependent losses, as shown in Fig. 9. The series resistances $R_{s 1}$ and $R_{s 2}$ are used to model the conductor loss dominant at lower frequencies, while the parallel and ground resistances $R_{p}$ and $R_{g}$ are used to model the substrate loss dominant at higher frequencies. It is without controversy that the $Q$ factor is the most representative parameter to evaluate the inductor loss. The most popular definition for the $Q$ factor is based on the one-port inductor model with the second port grounded, and can be generally expressed as the ratio of the imaginary to real part of the input impedance [7]-[10]. Such a $Q$-factor definition can be further derived as a function of the input reflection coefficient expressed in terms of $S$-parameters, which is given as

$$
Q=\frac{\operatorname{Im}\left\{Z_{\text {in }}\right\}}{\operatorname{Re}\left\{Z_{\text {in }}\right\}}=\frac{2 \operatorname{Im}\left\{\Gamma_{\text {in }}\right\}}{1-\left|\Gamma_{\text {in }}\right|^{2}}
$$

where

$$
\Gamma_{\text {in }}=S_{11}-\frac{S_{12} S_{21}}{1+S_{22}} .
$$

A simple optimization scheme for resistance values used in the modified-T and PI-section models has been developed on Agilent's Advanced Design System to fit the measured $Q$-factor response, as shown in Fig. 10. It is noted that the frequency range of interest in optimization is up to the first SRF where

$$
a=\frac{\omega_{p}^{2}\left(1-\frac{\omega_{p}^{2}}{\omega_{s 1}^{2}}\right)^{-1}-\omega_{p 1}^{2}\left(1-\frac{\omega_{p 1}^{2}}{\omega_{s 1}^{2}}\right)^{-1}}{\omega_{p}^{2}\left[\left(1-\frac{\omega_{p}^{2}}{\omega_{s 1}^{2}}\right)^{-1}-\left(1-\frac{\omega_{p}^{2}}{\omega_{s}^{2}}\right)^{-1}\right]-\omega_{p 1}^{2}\left[\left(1-\frac{\omega_{p 1}^{2}}{\omega_{s 1}^{2}}\right)^{-1}-\left(1-\frac{\omega_{p 1}^{2}}{\omega_{s}^{2}}\right)^{-1}\right]}
$$




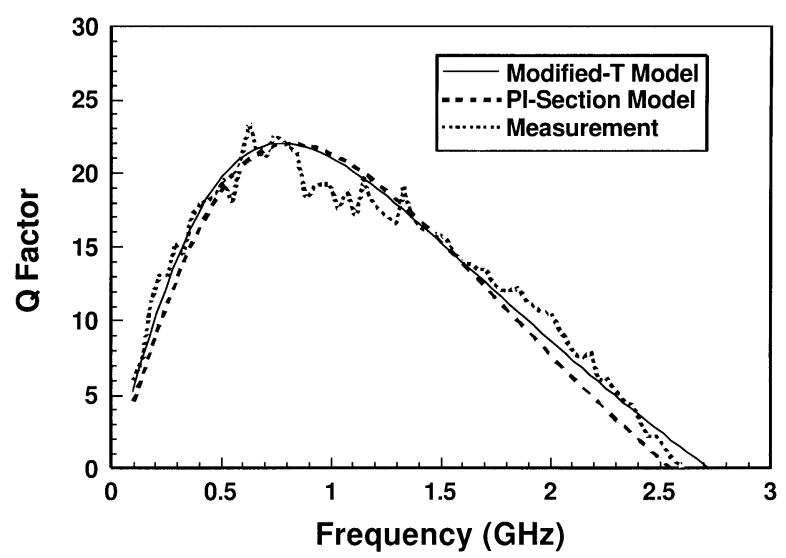

Fig. 10. Comparison of the $Q$ factor between the modified-T and PI-section models with measurements for a 6.8 -nH LTCC embedded spiral-type inductor.
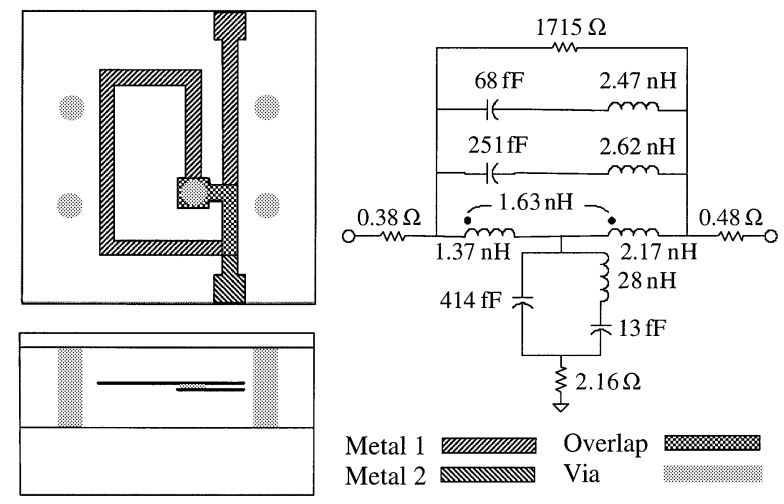

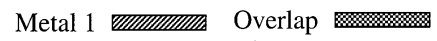
Metal 2 Via

Fig. 11. Top and side views of a 6.8-nH spiral-type inductor embedded in a multilayer LTCC substrate and the established equivalent modified-T model.

$Q$ factors are positive real numbers. Such an optimization takes only a few seconds using a standard PC. Within this frequency range, the measured and modeled $Q$ factors can agree quite well through optimization.

\section{EXAMPLES}

For demonstration, we have implemented three different types of embedded inductors including: 1) spiral; 2) meander; and 3) vertically stacked spiral in a 22-layer LTCC process. Each layer is 3-mil thick and the final substrate size is fixed at $120 \mathrm{mil} \times 120 \mathrm{mil} \times 66 \mathrm{mil}$. All inductors embedded in such an LTCC substrate are also designed with a fixed outer dimension of 120 mil $\times 60$ mil and have top and bottom ground planes for the purpose of suppressing electromagnetic interference (EMI). Both ground planes have a distance of 33 mil from one to the other and a total of four vias outside the inductor area to connect each other. The conductor width for all inductors is 6 mil and the substrate dielectric constant is 7.8.

Fig. 11 shows the geometry of a 1.5 -turn rectangular spiral inductor and the equivalent modified-T model. This device has an equivalent inductance of $6.8 \mathrm{nH}$ at low frequencies. All the reactive elements in the equivalent modified-T model can be found accurately using the analytical expressions provided in Steps 1-3 of the element-extraction procedure given in Section III. The resistances used to account for the frequency-dependent losses are found through optimization, as
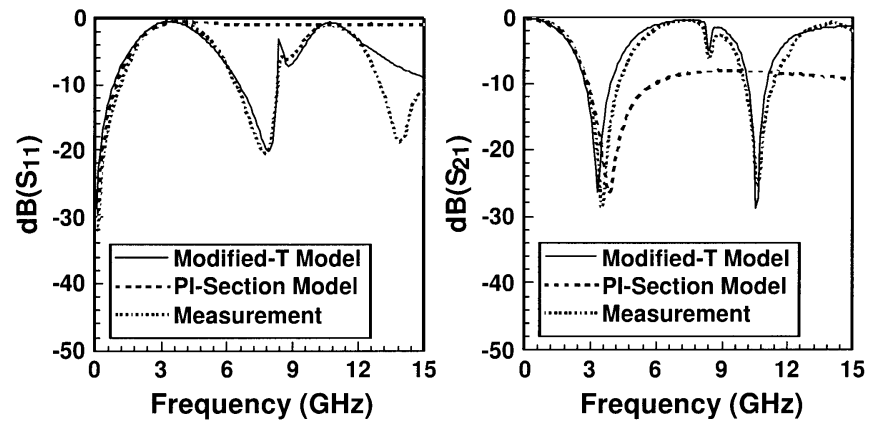

Fig. 12. Comparison of the magnitude of $S_{11}$ and $S_{21}$ in decibels between the equivalent modified-T and PI-section models with measurements for the 6.8-nH spiral-type inductor.
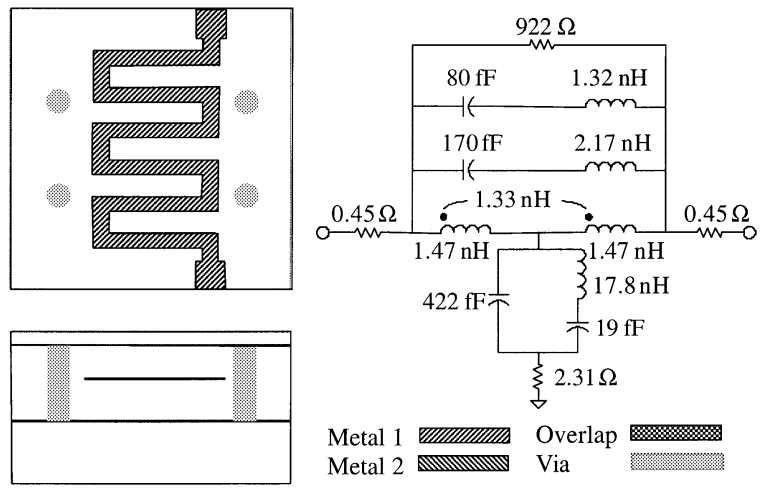

Fig. 13. Top and side views of a 5.6-nH meander-type inductor embedded in a multilayer LTCC substrate and the established equivalent modified-T model.

suggested in Step 4 of the procedure. In Fig. 12, we compare the $S$-parameter results generated from our proposed modified-T model and the conventional PI-section model with measurements within the frequency range up to $15 \mathrm{GHz}$. It can be seen that the conventional PI-section model is good only up to the fundamental SRF at $3.5 \mathrm{GHz}$. Our proposed modified-T model can catch not only the fundamental SRF, but also the GRF at $8.4 \mathrm{GHz}$ and the next higher SRF at $10.6 \mathrm{GHz}$. The effective bandwidth exceeds $12 \mathrm{GHz}$, approximately four times larger than in a PI-section model. As shown in Fig. 13, the case for a meander-type inductor having an equivalent inductance of $5.6 \mathrm{nH}$ at low frequencies can employ the same equivalent model structure. The modeled results shown in Fig. 14 also demonstrate that our proposed modified-T model surpasses the PI-section model quite obviously in comparison of bandwidth. Such a meander-type inductor has the fundamental SRF, GRF, and next higher SRF at 3.6, 8.4, and $12.9 \mathrm{GHz}$, respectively, which has been predicted successfully from the established modified-T model.

In the last example, shown in Fig. 15, two rectangular spiral inductors of opposite direction of rotation are stacked vertically. Each inductor has two turns of winding. The total inductance at low frequencies approaches $14.5 \mathrm{nH}$. Within the same frequency range, this inductor exhibits more SRFs and GRFs than the former two inductors. It is, therefore, required to expand in series and parallel feedback resonators of a modified-T model according to the increase of number of SRFs and GRFs, as also illustrated in Fig. 15. It is emphasized that all the reactive elements in this expanded modified-T model can still be found 

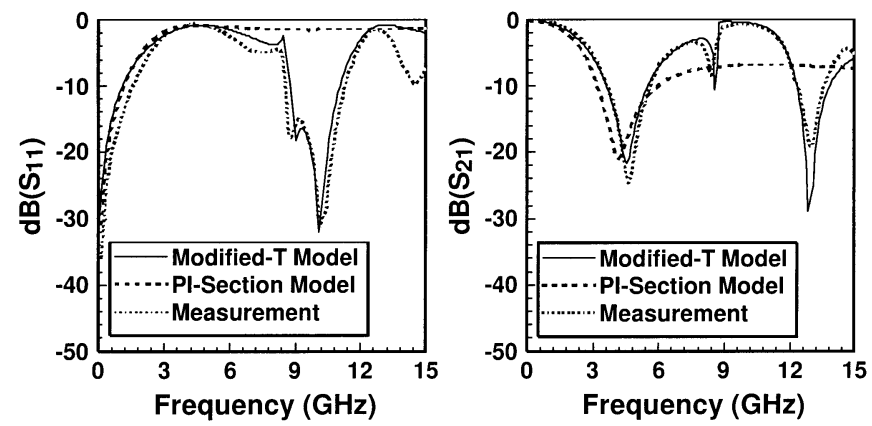

Fig. 14. Comparison of the magnitude of $S_{11}$ and $S_{21}$ in decibels between the equivalent modified-T and PI-section models with measurements for the 5.6-nH meander-type inductor.
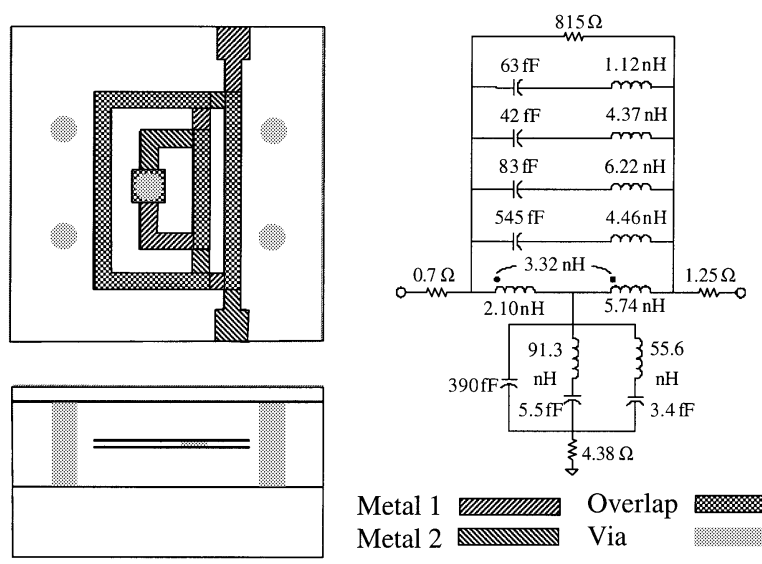

Metal 1 Metal 2 Via

Fig. 15. Top and side views of a 14.5 -nH vertically stacked spiral-type inductor embedded in a multilayer LTCC substrate and the established equivalent modified-T model.
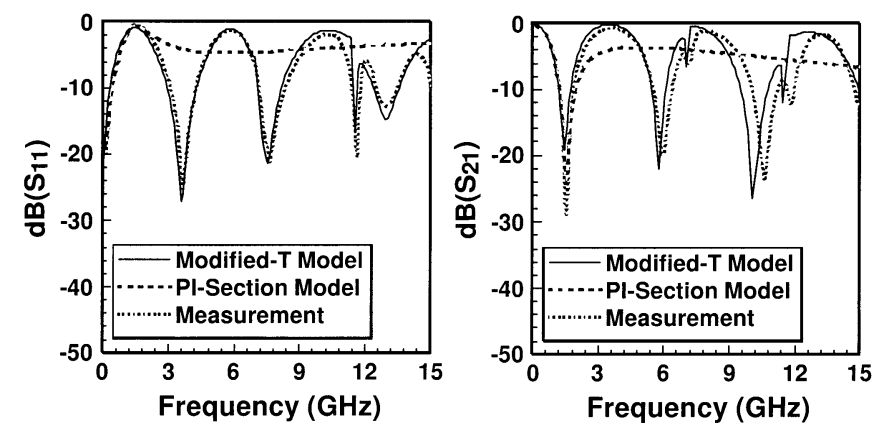

Fig. 16. Comparison of the magnitude of $S_{11}$ and $S_{21}$ in decibels between the equivalent modified-T and PI-section models with measurements for the 14.5-nH vertically stacked spiral-type inductor.

analytically with enough numbers of the identified SRFs and GRFs. Fig. 16 shows the comparison of modeled $S$-parameters with measured results. Again, only the modified-T model can provide good accuracy in the entire frequency range up to $15 \mathrm{GHz}$.

\section{CONCLUSION}

Due to the feature of broad-band transmission-line characteristics, the proposed modified-T equivalent circuit is successful in modeling a variety of types of LTCC embedded inductors with a bandwidth several times larger than a conventional

PI-section model. In addition, this new model structure can be constructed quite efficiently because all the reactive elements in the model can be extracted from the measured $S$-parameters analytically with the help of identifying SRFs and GRFs. The remaining few resistances in the model have been found through optimization to best fit the frequency-dependent $Q$ factor that can be also determined from the measured $S$-parameters. This modeling technique can be applied to any types of high-speed interconnects and should be helpful in analysis and design for compensating the discontinuity effects.

\section{REFERENCES}

[1] S. Chaki, S. Aono, N. Andoh, Y. Sasaki, N. Tanino, and O. Ishihara, "Experimental study on spiral inductors," in IEEE MTT-S Int. Microwave Symp. Dig., 1995, pp. 753-756.

[2] M. Rytivaara and F. L. Oy, "Buried passive elements manufactured in LTCC," in Proc. IEE Packaging and Interconnects at Microwave and Millimeter-Wave Frequencies Seminar, 2000, pp. 6/1-6/5

[3] A. Sutono, D. Heo, Y. E. Chen, and J. Laskar, "High- $Q$ LTCC-based passive library for wireless system-on-package (SOP) module development," IEEE Trans. Microwave Theory Tech., vol. 49, pp. 1715-1724, Oct. 2001.

[4] K. H. Drue, H. Thust, and J. Muller, "RF models of passive LTCC components in the lower gigahertz-range," Appl. Microwave and Wireless, pp. 26-35, Apr. 1998.

[5] S. Lee, J. Choi, G. S. May, and I. Yun, "Modeling and analysis of 3-D solenoid embedded inductors," IEEE Trans. Electron. Packag. Manufact., vol. 25, pp. 34-41, Jan. 2002.

[6] T. S. Horng, J. M. Wu, L. Q. Yang, and S. T. Fang, "A novel modified-T equivalent circuit for modeling LTCC embedded inductors with a large bandwidth," in IEEE MTT-S Int. Microwave Symp. Dig., 2003, pp. 1015-1018.

[7] C. P. Yue and S. S. Wong, "On-chip spiral inductors with patterned ground shields for Si-based RF IC's," IEEE J. Solid-State Circuits, vol. 33, pp. 743-752, May 1998.

[8] K. O., "Estimation methods for quality factors of inductors fabricated in silicon integrated circuit process technologies," IEEE J. Solid-State Circuits, vol. 33, pp. 1249-1252, Aug. 1998.

[9] A. Sutono, A. H. Pham, J. Laskar, and W. R. Smith, "RF/microwave characterization of multilayer ceramic-based MCM technology," IEEE Trans. Adv. Packag., vol. 22, pp. 326-331, Aug. 1999.

[10] H. Jiang, Y. Wang, J. A. Yeh, and N. C. Tien, "On-chip spiral inductors suspended over deep copper-lined cavities," IEEE Trans. Microwave Theory Tech., vol. 48, pp. 2415-2423, Dec. 2000.

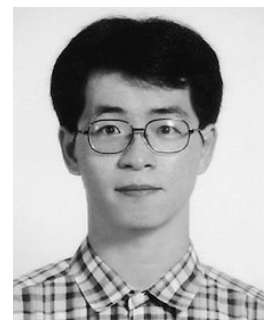

circuits and packages.

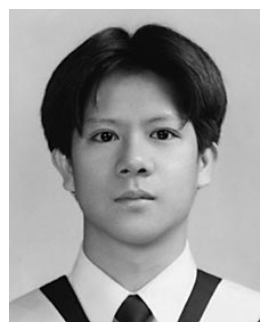

Tzyy-Sheng Horng (S'88-M'92) was born in Taichung, Taiwan, R.O.C., on December 7, 1963. He received the B.S.E.E. degree from the National Taiwan University, Taipei, Taiwan, R.O.C., in 1985, and the M.S.E.E. and Ph.D. degrees from the University of California at Los Angeles, in 1990 and 1992, respectively.

$\mathrm{He}$ is currently a Professor with the Department of Electrical Engineering, National Sun Yat-Sen University, Kaohsiung, Taiwan, R.O.C. His research interests are in the area of RF and microwave integrated

Jian-Ming Wu was born November 13, 1974, in Kaohsiung, Taiwan, R.O.C. He received the B.S.E.E. degree from the Yuan Ze University, Chungli, Taiwan, R.O.C., in 1997, the M.S.E.E. degree from the National Sun Yat-Sen University, Kaohsiung, Taiwan, R.O.C., in 2000, and is currently working toward the Ph.D. degree in electrical engineering at the National Sun Yat-Sen University.

His research interests include design and modeling of microwave integrated circuit components and packages. 


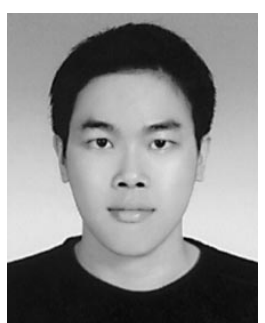

Li-Qun Yang was born October 20, 1978, in Tainan, Taiwan, R.O.C. He received the B.S. degree in communication engineering from the National Chiao Tung University, Hsinchu, Taiwan, R.O.C., in 2000 and the M.S.E.E. degree from the National Sun Yat-Sen University, Kaohsiung, Taiwan, R.O.C., in 2002.

$\mathrm{He}$ is currently an RF Engineer with the RF Communication Systems Technology Department, Computer and Communication Laboratories, Industrial Technology Research Institute (ITRI), Hsinchu, Taiwan, R.O.C. His research is focused on the design and modeling of LTCC embedded inductors and capacitors.

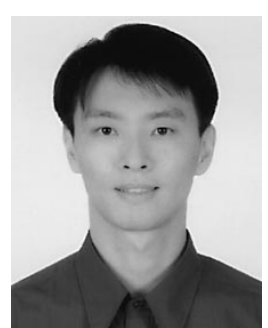

Shyh-Tirng Fang (M'99) was born in Tainan, Taiwan, R.O.C., in 1972. He received the B.Eng. degree in electrical engineering from the TamKang University, Taipei, Taiwan, R.O.C., in 1995, and the Ph.D. degree in electrical engineering from the National Sun Yat-Sen University, Kaohsiung, Taiwan, R.O.C., in 1999.

From 1995 to 1999, he was with the Electromagnetic Wave and Antennas Laboratory, National Sun Yat-Sen University, where he was a Research Assistant involved with the analysis and design of microstrip antennas. Since October 1999, he has been with the Computer and Communications Research Laboratories, Industrial Technology Research Institute, Hsinchu, Taiwan, R.O.C., where he is currently a Deputy Manager of the RF Communication Systems Technology Department. His research interest include antenna theory and design, propagation for wireless communications, and multilayer circuit designs.

Dr. Fang is a member of the IEEE Antennas and Propagation Society (IEEE AP-S), the IEEE Microwave Theory and Techniques Society (IEEE MTT-S), the IEEE Components, Packaging, and Manufacturing Technology (IEEE CPMT) Society, and the International Microelectronics and Packaging Society (IMAPS). He is also an honorary member of Phi Tau Phi. 